\title{
Pure arterial malformation of the posterior inferior cerebellar artery with interspersed adipose tissue: case report
}

\author{
Thomas J. Sorenson, BS, ${ }^{1}$ Waleed Brinjikji, MD, ${ }^{2}$ Kelly D. Flemming, MD, ${ }^{3}$ and \\ Giuseppe Lanzino, MD ${ }^{1,4}$
}

Departments of ${ }^{1}$ Neurologic Surgery, ${ }^{3}$ Neurology, and ${ }^{4}$ Radiology, Mayo Clinic, Rochester, Minnesota; and ${ }^{2}$ Department of Radiology, University of Toronto, Ontario, Canada

\begin{abstract}
Incidental vascular lesions are being discovered at an increasing frequency due to widespread noninvasive brain imaging studies. One of these lesions has recently been termed a "pure arterial malformation" (PAM), which is defined as dilated, overlapping, and tortuous arteries forming a mass of arterial loops with a coil-like appearance in the absence of arteriovenous shunting. The pathogenesis of these lesions is not known, but a congenital etiology is suspected. The authors report the case of a 17-year-old female who was found to have a PAM of the posterior inferior cerebellar artery with adipose tissue interspersed within the arterial loops. The authors believe that this abnormal intracranial association between blood vessel and adipose tissue lends further support to the theory that PAMs are the result of a congenital malformation and are therefore safe to manage conservatively given their presumed benign natural history. Far from offering conclusive evidence, this unique case report adds to the growing body of PAM literature and strengthens an increasingly supported congenital theory of genesis.
\end{abstract}

https://thejns.org/doi/abs/10.3171/2018.4.PEDS18135

KEYWORDS pure arterial formation; posterior inferior cerebellar artery; magnetic resonance imaging; pineal cyst; catheter angiography; incidental; vascular disorders; congenital

$\mathrm{W}$ ITH the widespread utilization of noninvasive imaging studies, incidental vascular lesions are discovered and diagnosed with increasing frequency. In the past few years, a newly described vascular pathology has been characterized by the sole presence of coiled, sometimes ectatic, arterial loops without any arteriovenous shunting. These lesions have been termed "pure arterial malformations" (PAMs), ${ }^{4,5}$ Presently, the genesis and clinical significance of PAMs are unresolved. One of the initial hypotheses was that these lesions could represent asymptomatic, healed arterial dissections. However, recent cases and the benign natural history of PAMs have raised an alternative hypothesis about a possible congenital nature. ${ }^{4}$

Herein, we describe a patient with an intracranial PAM of the posterior inferior cerebellar artery (PICA) with interspersed adipose tissue. We believe, based on embryological considerations, that this association between blood vessel and adipose tissue could lend further support to the hypothesized congenital nature of PAMs as developmental anomalies.

\section{Case Report}

History and Examination

A 17-year-old female underwent brain imaging because of migraines with aura. Coiled loops of the proximal PICA were noted on MRI (Fig. 1A). This was considered an incidental finding, and no further investigation or intervention was recommended. The headaches were well controlled 

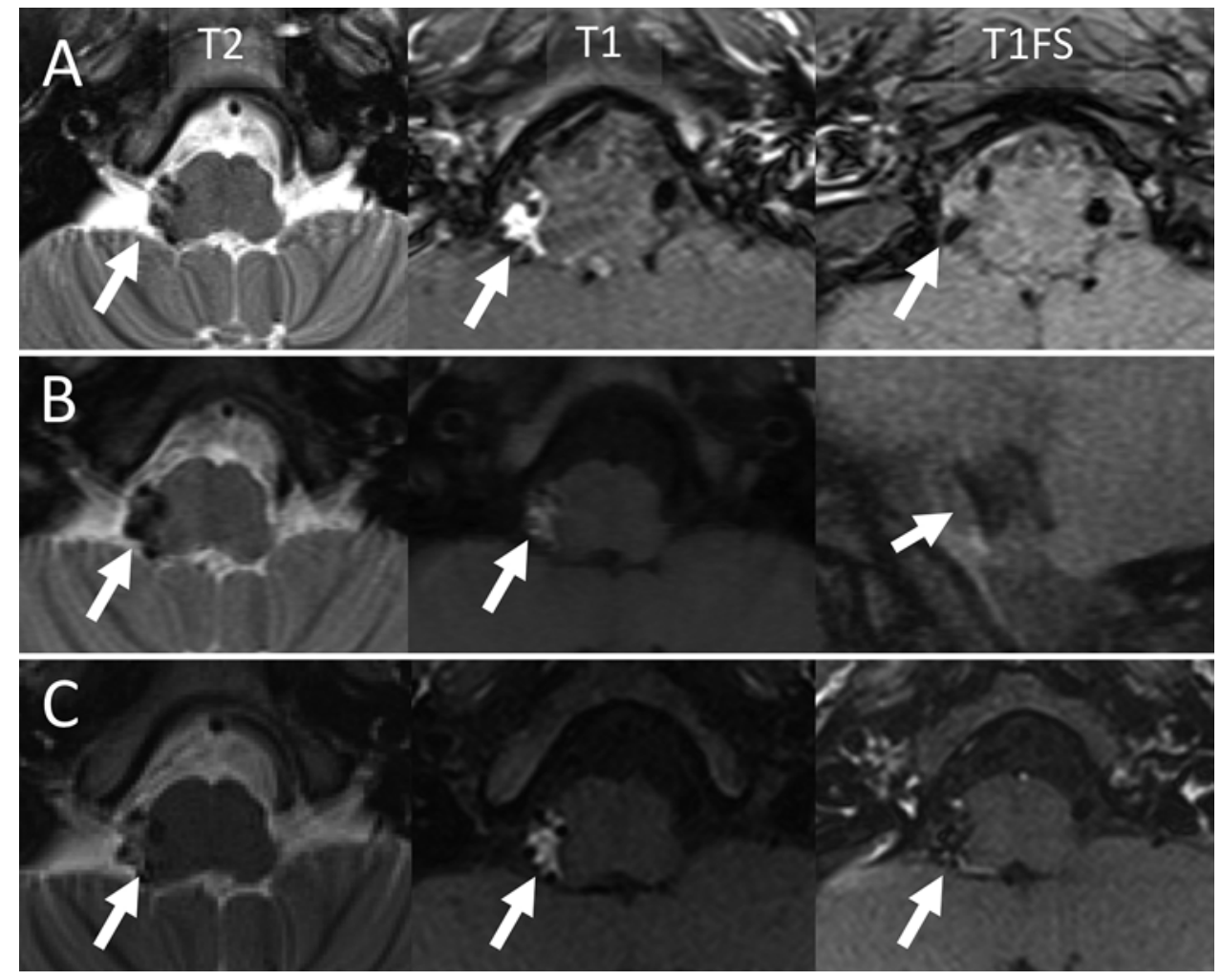

FIG. 1. Serial follow-up over 8 years of the PAM. A: Baseline axial T2-weighted, T1-weighted, and T1 fat saturated images show a fat-containing lesion with enlarged vascular flow voids in the right perimedullary space (arrows). B: Five-year follow-up MRI demonstrated no change in the appearance or size of the fat-containing lesion with flow voids. C: Eight-year follow-up MRI demonstrated no change.

for 4 years, after which she noticed an increased frequency of migraines and associated visual disturbances. This change prompted repeat imaging, and MRI showed the previously discovered anomaly to be stable (Fig. 1B). Because of the increasing severity and frequency of her symptoms at the age of 25 years, she was referred to our institution in the hopes of determining a possible association between the noted vascular anomaly and her symptoms. We repeated MRI of the brain, which again showed an abnormal, but stable, vascular structure. The T1 hyperintensities interspersed within the coiled loops of the right PICA were shown to be fat elements on baseline MRI with T1 and fat-suppression sequences (Fig. 1C) and on head CT (Fig. 2). Catheter angiography confirmed the coiled loops of the proximal PICA without arteriovenous shunting, consistent with a PAM. The dysplastic nature of these loops was suggested by the increased diameter of the PICA vessel located distal to the PAM (Fig. 3). The lesion was not believed to be an angiolipoma since angiolipomas characteristically demonstrate blush from enlarged capillaries and venules rather than enlarged, tortuous, and dilated large and medium-sized arteries.

\section{Treatment}

Given these imaging findings, we believed the constellation of symptoms was in no way related to the anomalies in question and the PAM was incidental. Thus, no further treatment besides medical management of her headaches was recommended.

\section{Discussion}

We report the case of a patient with an intracranial PAM of the proximal PICA with adipose tissue interspersed between the coiled vessel loops. This was an incidental finding on MRI that was stable over 8 years. Though the first official characterization of PAM was published by McLaughlin et al. in 2013, ${ }^{5}$ upon retrospective analysis,

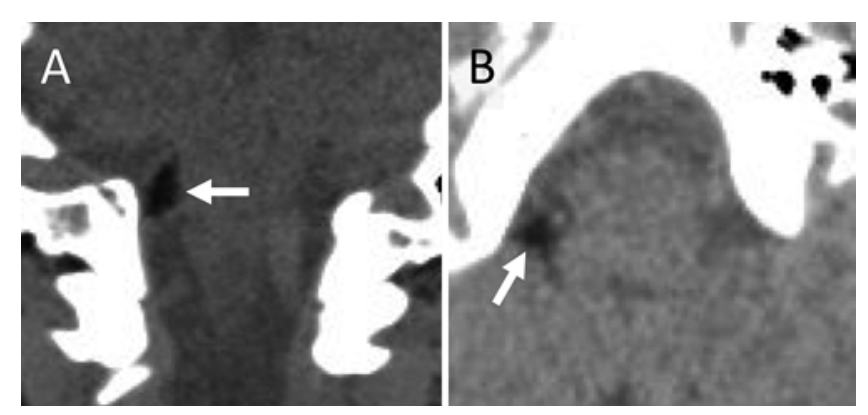

FIG. 2. Noncontrast CT of the PAM in coronal (A) and axial (B) planes demonstrated macroscopic fat in the right perimedullary space (arrows), thus confirming that the vascular loops were interspersed with adipose tissue. 

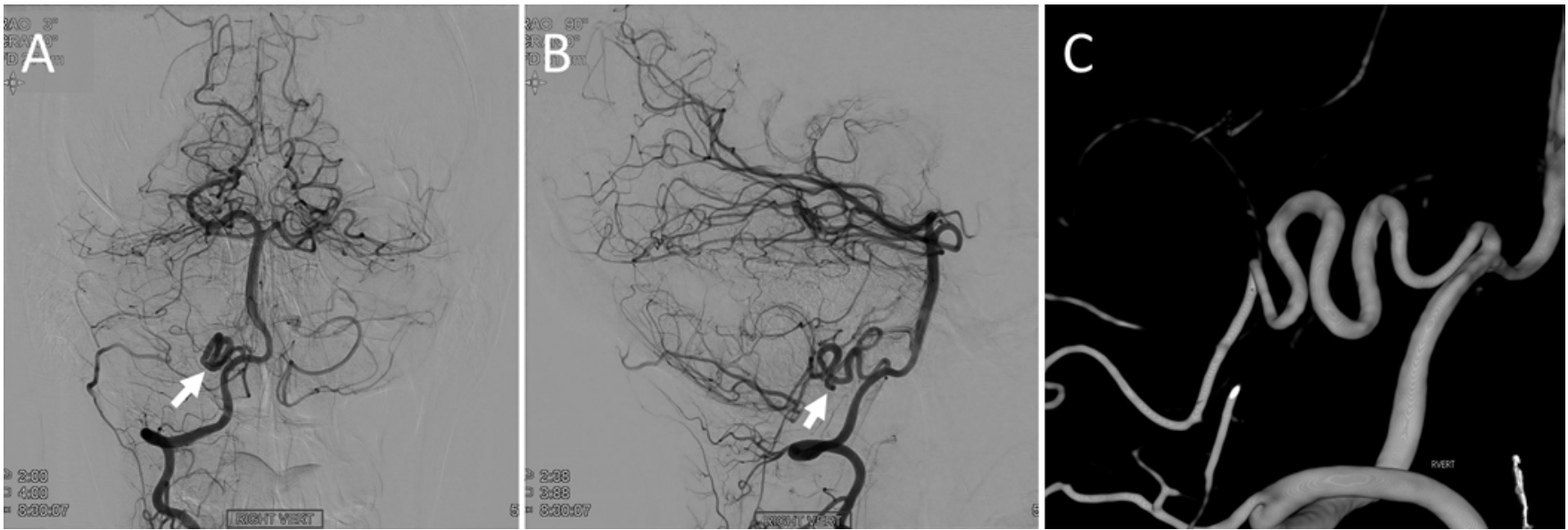

FIG. 3. Anteroposterior digital subtraction angiography (DSA; A), lateral DSA (B), and 3D rotational angiography (C) obtained after right vertebral artery injection, demonstrating the PAM of the right PICA, which has a coil-like configuration in its proximal portion and is ectatic as well (arrows). There is no blush of the adipose tissue as would be characteristically observed in an angiolipoma.

we identified at least 20 other individual case reports that meet the "McLaughlin criteria" in the literature. ${ }^{4,5}$ Despite the growing volume of confirmed PAM cases, their mechanism of genesis is still unknown.

Many potential etiologies have been hypothesized for these lesions, including arterial dysplasia resulting from a congenital defect, chronic healed dissection, and an insult such as a viral infection or somatic mutation occurring later in life and affecting a particularly vulnerable arterial segment (i.e., segmental arterial vulnerability). Our theory of arterial dysplasia resulting from a congenital defect is supported by the current case report and several other reported cases, such as case 8 in the Brinjikji et al. series, which demonstrated cortical or white matter dysplasia in the supplied area of the PAM. ${ }^{1-4,6,9}$ We believe that the patient in the current report with a PAM of the proximal PICA with fat elements interspersed among the coiled arterial loops supports the developmental congenital theory.

The association between proximal PICA loops and adipose tissue elements could be explained based on embryological considerations. The first precursors of the CNS develop during the 4th week of embryological development from the neural tube, including the rhombencephalon, which develops into the metencephalon, the cerebellum precursor. Alongside this development, the cerebrovasculature develops from an original perineural vascular plexus (PNVP) that is located outside the CNS and extends alongside the growing brain structure. ${ }^{8}$ In our case, the proximal portion of the PICA, which supplies a phylogenetically early portion of the cerebellum (archicerebellum) and where we observed the anomaly, develops early as a superior branch of the spinal artery, whereas the distal portion, which supplies a relatively more recent portion of the cerebellum, develops much later. In our patient, during these early phases of brain development, mesoderm germ layer cells (which become both adipose tissue and blood vessels) could differentiate into the corresponding "downstream" tissue incorrectly near the origin of the PICA, leading to a blood vessel (PICA) with interspersed adipose tissue, with the adipose tissue potentially even guiding the PICA loops around it. This could explain the PAM with the unlikely coexistence of the loops of the proximal PICA and the adipose tissue that we saw in our patient.

Prior to the recognition of PAM as a distinct entity, two previously reported cases identified PAM-like lesions associated with intracranial lipoma. In a 1972 case series of angiographic findings associated with lipomas of the corpus callosum, Wolpert et al. reported three cases in which the pericallosal arteries were ectatic and tortuous, closely resembling the appearance of distal anterior cerebral artery PAMs described in the Brinjikji et al. series. ${ }^{4,9}$ More recently, in 2000, Saatci et al. reported the case of a sylvian fissure lipoma associated with cortical dysplasia and an abnormally dilated and tortuous distal middle cerebral artery branch that had the classic coil configuration seen in PAMs inside the lipoma. ${ }^{7}$ Such observations are important as they strengthen the evidence for the benign natural history of these lesions. Recognizing that these lesions are incidental, likely as a stable remnant from the beginning of embryonic development, can provide guidance for clinical decision-making. As developmental anomalies have benign natural histories, patients with incidentally discovered PAMs can be safely treated with medical management of their presenting symptoms and avoidance of unnecessary complicated and risky procedures.

\section{References}

1. Abe T, Singer RJ, Marks MP, Kojima K, Watanabe M, Uchida M, et al: Arterial vascular abnormality accompanying cerebral cortical dysplasia. AJNR Am J Neuroradiol 18:144-146, 1997

2. Araki Y, Mori S, Kanoh M, Maeda Y, Kawai R, Mitomo M: Congenital hemicerebral arterial ectasia complicating unilateral megalencephaly. Br J Radiol 60:395-400, 1987

3. Baccin CE, Krings T, Alvarez H, Ozanne A, Lasjaunias PL: A report of two cases with dolichosegmental intracranial arteries as a new feature of PHACES syndrome. Childs Nerv Syst 23:559-567, 2007

4. Brinjikji W, Cloft HJ, Flemming KD, Comelli S, Lanzino G: 
Pure arterial malformations. J Neurosurg [epub ahead of print September 29, 2017; DOI: 10.3171/2017.2.JNS1744]

5. McLaughlin N, Raychev R, Duckwiler G, Martin NA: Pure arterial malformation of the posterior cerebral artery: importance of its recognition. J Neurosurg 119:655-660, 2013

6. Metry DW, Dowd CF, Barkovich AJ, Frieden IJ: The many faces of PHACE syndrome. J Pediatr 139:117-123, 2001

7. Saatci I, Aslan C, Renda Y, Besim A: Parietal lipoma associated with cortical dysplasia and abnormal vasculature: case report and review of the literature. AJNR Am J Neuroradiol 21:1718-1721, 2000

8. Tata M, Ruhrberg C, Fantin A: Vascularisation of the central nervous system. Mech Dev 138:26-36, 2015

9. Wolpert SM, Carter BL, Ferris EJ: Lipomas of the corpus callosum. An angiographic analysis. Am J Roentgenol Radium Ther Nucl Med 115:92-99, 1972

\section{Disclosures}

The authors report no conflict of interest concerning the materials or methods used in this study or the findings specified in this paper.

\section{Author Contributions}

Conception and design: all authors. Acquisition of data: all authors. Analysis and interpretation of data: all authors. Drafting the article: all authors. Critically revising the article: all authors. Reviewed submitted version of manuscript: all authors.

\section{Correspondence}

Giuseppe Lanzino: Mayo Clinic, Rochester, MN. lanzino. giuseppe@mayo.edu. 